\title{
COMPUTATIONAL TRUST MANAGEMENT IN ECONOMICS PHENOMENA RESEARCH
}

\author{
Denis TRČEK ${ }^{*}$ \\ Department of Informatics, Faculty of Computer and Information Systems, \\ University of Ljubljana Večna pot 113, 1000, Ljubljana, Slovenia
}

Received 25 September 2015; accepted 25 June 2017

\begin{abstract}
Economics phenomena are notably governed by dynamic, non-linear, bottom-up processes emerging from agents' interactions. Therefore traditional top-down approaches provide a rather limited insight into these phenomena. Further, research in economics has been mostly focused on addressing tangible factors, while human agents in economic settings often do not adhere to rational reasoning, and trust is one such kind of reasoning. Thanks to recent technological advancements new approaches are enabled, and this paper proposes a novel and anticipatory research methodology for studying economics phenomena that enables inclusion of trust. The methodology, called auxiliary composite simulations, builds upon recent advancements in computational trust management. By doing so it enables bottom-up simulations of trust driven economic phenomena. The paper provides also epistemic evaluation of the methodology and ends up with an example application of the proposed apparatus.
\end{abstract}

Keywords: economics research, agent technologies, trust, computational trust management, simulations.

JEL Classification: C63, R10.

\section{Introduction}

Economic systems are non-linear dynamic structures. They consist of atomic entities, i.e. human agents, their initial interactions, and from these two constituents emerging aggregates, i.e. new composite agents and new composite interactions structures. While traditional approaches to economics have often idealized humans as rational agents, research in the last few decades proved otherwise. One such example is Prospect theory by Kahneman and Tversky (1979).

A long time dominating paradigm of rational agents is just one side of the coin. The other side is that traditional theories in economics are being of a top-down nature. Various phenomena can be researched this way, but it is obvious that there are many examples that

*Corresponding author. E-mail: denis.trcek@fri.uni-li.si

This is an Open Access article distributed under the terms of the Creative Commons Attribution License (http://creativecommons. org/licenses/by/4.0/), which permits unrestricted use, distribution, and reproduction in any medium, provided the original author and source are credited. 
are naturally evolving bottom-up. Their addressing has therefore to be bottom-up as well, which is becoming feasible with the latest technological advancements.

This is also where the main contributions of this research paper come in. The first is inclusion of trust in economics research through computational trust management. Trust is one key non-rational factor that is driving a large part of economic activities, while being rather unresearched (with some exception being experimental economics). The second contribution is focus on bottom-up analyses through agents based modelling, ABM. Both contributions are enabling so called auxiliary composite simulations. This paper also demonstrates the application of this method to spatial agglomerations studied through trust and bottom-up approaches with ABM.

The paper is structured as follows. In section two an overview of the literature is given that focuses on economics, ABM, trust, and agglomerations research. In section three epistemic views on inclusion of trust in economics phenomena research are given. This is followed by a section that presents the details of auxiliary composite simulations linked to computational trust management method called Qualitative Assessment Dynamics, QAD (Trček 2012; Zupančič, Trček 2017). Next comes demonstration of the presented apparatus with focus on agglomerations research. The paper ends with conclusions, acknowledgements and references.

\section{An overview of relevant research in economics}

The first subsection below covers the literature overview of traditional economics research (including agglomerations), while the second covers ABM based research. The presented papers are evaluated through perspective of trust.

\subsection{Traditional economics research and trust}

Recent global financial crisis has challenged quite some premises of traditional economics. In Trichet (2010) it is urged that the characteristics of homo economicus have to be rethought. Seeing economics as a sum of atomic optimization searching mass of rational agents seems to be getting to its limits. This kind of treatment prevents seeing heterogeneity across agents and limits us in seeing (only) decisions that drive the system as a kind of optimization problem. Therefore the observed processes have not been covered adequately, as they are, in many cases, a result of richer characteristics behind them. We are not considering here only such factors like rational inattention or bounded rationality, where the decisions of agents are hampered by limited information, time availability, or processing capability, but also such factors like trust.

In Cox et al. (2008) the authors challenge the paradigm of rational homo economicus by studying actions, which are inconsistent with this paradigm. Their experiments support claims that human behavior is characterized by trust. It is shown that trust is inherently endogenous to humans when it comes to positive reciprocity. One earlier research also indicates the correctness of this statement by finding that trust is based on beliefs of trustworthiness and on unconditional kindness (Ashraf et al. 2006). These beliefs and unconditional kindness 
go to such extent that people largely exclude potential monetary benefits: "People trust even though hardly anyone makes money by doing so." And people are aware of this ${ }^{1}$.

In Song (2006) Trust game is deployed to examine whether the behavior of group representatives differs from those of the same individuals when acting on their own. The result is that trust in inter-individual interactions differs from inter-group interactions. Interestingly, group representatives trust less when acting on behalf of a group than when acting on their own. Further, when groups make consensus their level of trust does not differ from that of individuals, while reciprocity dramatically decreases. Therefore one cannot make conclusions on group trust by inferring from individual counterparts (the same holds true for reciprocity). As to external factors, i.e. those that make influence from the outside, the research in Schotter, Sopher (2006) exposes recommendations. Using Trust game it is found out that people change their trust when facing a certain situation if they are given an advice. Another external factor is heterogeneity. In Anderson et al. (2006) a frequent claim is explored that heterogeneity among individuals reduces trust. These authors also use Trust game and induce non-homogeneity by varying show-up payments that are given to subjects for participation. It has been found out that heterogeneity does not influence trust, which contrasts other research in this area where results typically indicate that heterogeneity does hinder trust. One well-known such work in the area of social capital is Putnam (1995).

In Kurzban et al. (2008) a practical paradigm is investigated, which is how trust can be built up. For these experiments the original Trust game has been slightly modified to allow only increasing incremental investments in investigated subjects' counterparts. The results suggest that people prefer building up trust gradually, in small increments, rather than following "one big shot" moves".

Interesting research exists also about relations between trust and competition. While the majority of research shows that competition is not a good humus for growth of trust (see, e.g. Tjosvold 1985; Ferrin, Dirks 2003), there exist some evidence that the contrary may be true. In Keck, Karelaia (2012) it is suggested that competition positively affects trust not only within dyads of direct competitors, but also in dyads of individuals who do not compete against each other. It is worth to add that trust and reciprocity are likely to be behavioral instincts. Many research examples confirm that even differences in information presentation and strategic reasoning prompts did not change the "basic instincts" (Ortmann et al. 2000).

Let us shift now our focus from trust to agglomerations research. The parallel developments of economics of agglomeration and economics of networks are considered by Johans-

\footnotetext{
${ }^{1}$ Caveat - when it comes to trust, researchers are often applying experiments aimed at measuring behavior related to monetary gains, but trust has often little to do with monetary gains.

2 Apropos Trust game - the above given overview of related works shows the importance of Trust game for researching trust in economics. This game has been used in numerous economics contexts. On one side of the spectrum it has served to provide insights into how transactions could be influenced by merely including articulatory fluency (Zürn, Topolinski, 2017). More precisely, mere ease of some stimulus processing often affects intuitive judgments, e.g. when trustees carry real fluent surnames or artificial names they are trusted more. On the other side of the spectrum the effects of punishment on trust are studied using this game. In Calabuig et al. (2016) it is shown that, e.g. the difference between the investor and the allocator's initial endowments determines the effect of punishment on trust (and trustworthiness in general). These two examples demonstrate the richness and variety of implicit assumptions and understandings in the background of using Trust game for measuring trust. They further demonstrate how elusive phenomenon trust is.
} 
son and Quigly in Johansson, Quigley (2004). In their focus there are complementarities between the agglomeration productivity benefits and network linkages benefits. An interesting consequence of this analysis is that networks of agents dispersed over an area may be a substitute for their agglomerations at some location. Although this paper is not explicitly touching trust issues, its basic idea is very much related to that of invoking trust in agglomerations research through simulations. Further, in Rosenthal, Strange (2008) authors study estimations about the relationship of agglomeration and proximity to human capital (and to wages), while also focusing on trust through the attenuation of agglomeration and human capital effects, which attenuate sharply with distance.

\subsection{ABM based research}

Quite some areas of economics have been covered by ABM so far and this demonstrates the importance of bottom-up approaches. To start with equilibrium focused questions of classical and neoclassical economics - the core question of what actions of agents lead to equilibrium is addressed in Arthur et al. (1996). A decade later these authors extended the focus by examining the behavior of an economy when it is out of equilibrium (Arthur 2006). Their message is that out-of-equilibrium problem is not just a minor adjunct to traditional economics. It actually means a holistic addressing of economics. Consequently, this improves two indeterminacies of traditional approaches by being able to resolve among multiple equilibria and by modelling individuals' choices of expectations.

$\mathrm{ABM}$ based research soon turned to more sophisticated factors, where some pioneering research about transactions costs was done by Klos and Nooteboom in 2001 (Klos, Nooteboom 2001). As opposed to traditional transaction cost economics that frequently neglects learning, ignores the complexity of agents' landscape, assumes the efficient outcomes, and overlooks the development of trust, this work takes all the above mentioned factors into account. The model presents co-operation with trust emergence and is one rare example of existing $A B M$ research, where trust is explicitly addressed.

A niche oriented research followed recently, e.g. in industrial management and labor economics. One seminal work in this area (Leombruni, Richiardi 2003) deals with typical questions like how that individual "disorder" often leads to aggregate regularities, or, how individual "order" often leads to aggregates without regularity. These questions are rather frequent in economics, but in this case they are focused on particular areas such as power grids and energy market (Koesrindartoto et al. 2005). The systems in these latter areas have hard limits of distributed physical components that are restricting behaviors of distributed participating agents. This leads to specific dynamic performance of such structures, and ABM can address it naturally.

An interesting approach is given in Axtell (2005) where market mechanisms are compared with respect to their computational complexity. The first is the conventional Walrasian interpretation (where prices are centrally computed by an auctioneer), while the second is following a decentralized concept where concurrent exchange within agents in transient coalitions is taking place ( $\mathrm{k}$-lateral exchange). The main message of this paper is advocating for $\mathrm{k}$-lateral markets approaches. This is not only due to their closer reflection of reality, but also 
for practical reasons. Studying exchange equilibria with the latter model requires significantly less computing power. It is in principle polynomially bound, while in the former case, and in the worst case, it is even exponential with respect to number of commodities $\mathrm{N}$. This paper is somehow departing from traditional paths of economics research by addressing computational complexity, and paving the path for methodological approaches of such kind that are at the core of this paper.

Recently, also ABM based research endeavors appeared that explicitly address trust. In Barrio et al. (2017) a dynamical model for describing trading interactions between agents is studied with two key factors in focus being price and trust. Agents are able to sell and buy goods, where the decision to do so depends on the level of trust the buyer has in the seller, the price of the goods and the amount of money and goods that the buyer has at the disposal. By taking into account the whole trading history the model reveals the surprising effects that trust plays in economic interactions: it not only makes trading relations more robust, but also the wealth distribution in a society becomes more even.

Finally, one particularly important area in economics that has gained momentum recently - probably due to the personal interest of P. Krugman - is deployment of ABM in geographical economics. The first attempts can be found in Kimura (2002), where ABM simulations serve for getting insights into agglomerations.

The areas mentioned above are clearly not the only ones when it comes to ABM in economics, but they are most closely related to the focus of this paper.

\section{Epistemic issues related to trust research in economics}

This section elaborates epistemic issues related to concrete inclusion of trust in economics phenomena research, in particular in agglomerations studies.

Previous section shows that trust in economics is still a rather unexplored area. To start addressing it, the initial problem is the very definition of trust. In social sciences its understanding is often implicit, informal, and becomes precise when the context of its use is taken into account. According to Cambridge dictionary, trust is "belief that someone is good and honest and will not harm you, or that something is safe and reliable". This definition is still implicit if we are concerned with the very core of trust phenomenon. Trust is about reasoning processes in human brain, i.e. processes in (neo)cortical and sub-cortical parts of our brain Pessoa (2008). Therefore to explicitly and quantitatively tackle it at its core the neuro-science based methods could provide the basis through electro encephalography, functional nuclear magnetic resonance, functional near infrared spectroscopy and polygraphy (Trček 2012). However, to the best of our knowledge, there exists no quantitative definition based on the above approaches. But from the epistemic point of view this does not prevent us to study trust in the field of economics. Based on research of various phenomena in many scientific disciplines it is legitimate to proceed with treating it at "its face value" with the approach known as "black-box approach".

The next issue is what kind of scale for expressing trust should be deployed. It is sensible to use such scale that most humans are familiar with, and use it already in one way or another. The extensive review of the literature reveals that trust is often measured by categori- 
cal assessments like "trusted" and "distrusted". This two categorical values scale is frequently extended to semantic differential scales that range from three levels (i.e. trusted, undecided, distrusted) to five levels (i.e. totally trusted, partially trusted, undecided, partially distrusted, totally distrusted).

Considering more than five levels, some piloting research suggests that this would excessive (Trček 2011). Therefore we advocate for five-levels scale. Focusing now on agglomerations research, this research identifies two kinds of critical factors related to agglomerations, non-tangible and tangible factors:

- Non-tangible factors came into focus during recent decades. The majority of them is concentrated on social capital, which is defined as "networks together with shared norms, values and understandings that facilitate co-operation within or among groups" (Keeley 2007). The same author states further that "these networks and understandings engender trust and so enable people to work together."

- Tangible factors are those that are most extensively researched, and they can be considered as a kind of a main-stream research approach in agglomerations area. They are covering traditional "hard" factors like lower costs, spill-overs, and so on.

The research done by Borje and Quigly indicates that "market agents can increasingly substitute agglomeration proximity for network contacts and vice versa" (Johansson, Quigly 2004). This statement points towards a strong relationship between physical agglomerations and non-tangible substitutes, i.e. networks.

The problem is now how to link trust to tangible factors, and this will be addressed in this section in detail. We will stick with the basics of regional economics (agglomerations dynamics) and start with its antecedents (Fujita et al. 1999):

- The first is location theory that has at least three streams - one was following the von Thuenen analysis of land rent and land use, the second is Weber's stream that is focused on optimal plant location, and the third is the Central-place theory of Christaller and Loesch that builds on trade-off between scale economies and transportation costs. For the formal presentation the first stream is chosen: $R=Y(\rho-\gamma)-Y F m$, where $R$ stands for land rate, $Y$ for yield per land unit, $\gamma$ for production expenses per commodity unit, $\rho$ for market price of a commodity, $F$ for freight rate and $m$ for distance to market.

- Another building block is Base-Multiplier Analysis where export activities (of a region, state, etc.) are what their (economic) existence is about (also referred to as base activities), while non-base activities are derived from the base activities and they grow or shrink in line with performance of base activities. Formally, let $X$ be the income of a region due to its export, and let us treat it as exogenous variable. Further, let a constant fraction be spent locally on non-base products. Then direct earnings $X$ from exports lead to the next round of earnings $a X$, which in turn generates another round when spent locally, $a^{2} X$ and so on. As turns go to infinity, the resulting geometric series presents regional income $Y=X /(1-a)$. Now as the economy of the observed region rises, so does the fraction $a$, resulting in rising $Y$.

- An often used model is the market potential analysis, where market potential function $M$ typically measures the potential of an observed site $r$ as a weighted sum of 
the purchasing power of all other sites $s$, where weights are declining with a distance. Purchasing power is weighted inversely to distance, therefore $M_{r}=\Sigma_{s}\left(P_{s} / D_{r s}\right)$, where $D_{r s}$ is the distance from $r$ to $s$, and $P_{s}$ is the purchasing power at $s$.

By analyzing the above antecedents (and leaving more complex models like Dixit-Stiglitz model aside) it turns out that sensible inclusion of trust is not a trivial issue. These equations contain aggregate variables, which emerge as a consequence of agents' interactions, where these interactions are not analytically visible even implicitly. Therefore the following, orthogonality principle, is proposed:

- In cases where trust can be treated as being independent of variables used in the above models (equations), it will be superimposed on the results of agglomerations models.

- In cases when trust is not independent of variables in agglomerations models, such intervals have to be identified where the involved variables can be considered as being trust-independent, and superimposed accordingly.

When the orthogonality principle is fulfilled, ABM serves to obtain the spatial and temporal dynamics of trust first. Independently from this dynamics, appropriate model like one from those mentioned above is used to obtain spatial and temporal dynamics of the observed regional phenomenon, i.e. particular agglomeration. Finally, both models are processed on the basis of appropriately defined function, called trust fusion function. The auxiliary composite simulation method can be now summarized as follows:

1. Select appropriate regional phenomenon model and obtain its spatial and temporal results $p=p(x, y, t)$, where $p$ stands for the regional phenomenon, while $x$ and $y$ are coordinates, and $t$ denotes time.

2. Select appropriate computational trust management model and run it to get the spatial and temporal dynamics of trust. Therefore $\alpha=\alpha(x, y, t)$ is the result of this step, where $\alpha$ stands for trust value, $x$ and $y$ are coordinates, and where $t$ denotes time.

3. Define the fusion function $f$ that operates on inputs $p=p(x, y, t)$ and $\alpha=\alpha(x, y$, $t$ ), and maps these inputs to an output $c=f(p, \alpha)$. This fused value presents spatial phenomenon adjusted dynamics function due to effects of trust.

The nature of fusion function depends on the concrete research problem at hand. In case of a trust methodology having only two values ("trusted" and "distrusted") it can provide results as they would be without this function when $\alpha=$ "trusted", i.e. $c=p$, while when $\alpha=$ "distrusted" the composite result would be $c=0$ regardless of $p$. Further, when semantic differential scale values are used (e.g. "totally trusted", "partially trusted", "undecided", "partially distrusted", "totally distrusted"), the corresponding $p$ values in regions can be weighted according to these values by using weights like $(1,0.75,0.5,0.25,0)$, respectively. So if $\alpha=$ "partially trusted", then $c=0.75 p$.

\section{Auxiliary composite simulations}

Auxiliary composite simulations are simulations that implement the epistemic approach presented in the previous section. Two (or more) interfering phenomena, which are researched, are each modelled independently with a corresponding ABM method. After obtaining the partial results with these methods, the final, composite result is derived by using appropriate fusion function, if the conditions mentioned above are met. 
Agglomerations models are established, well elaborated area, and will not be discussed in detail in this paper - just a particular output agglomeration of such model will be assumed. The situation is different with computational trust management, which will be covered in more detail next. This should enable the reader to understand how trust dynamics in a particular society evolves, and how it is consequently used in our apparatus.

\subsection{Qualitative Assessment Dynamics - QAD}

To introduce computational trust management QAD is chosen. The first reason is that it is, to the best of our knowledge, the only anthropocentric computational trust management method. Next, it is performing well compared to other computational trust management methods (Jelenc et al. 2013). Finally, its potential for applications in decision making and e-commerce is covered, too Trček (2014) and Zupančič, Trček (2017).

To get the basic familiarity with QAD, the following definitions have to be given first:

Definition 1. Trust between agents A and B is a relation denoted as $\alpha_{A, B}$, which means agent's A trust assessment about agent $\mathrm{B}$.

Definition 2. In a society with $n$ agents an assessment matrix $\mathbf{A}$ is formed that consists of values $\alpha_{i, j}, i=1,2, \ldots, n$ and $j=1,2, \ldots, n$, where the values of $\alpha_{i, j}$ can be $-2,-1,0,1$, or 2 , meaning totally distrusted relation, partially distrusted relation, undecided relation, partially trusted relation and totally trusted relation. In cases where $\alpha_{i, j}$ does not exist, or cannot be obtained, it is denoted by “-”.

Definition 3. Each agents society has associated a dependency matrix $\Xi$ with ponders $\xi_{i, j}(i=$ $1,2, \ldots, n$ and $j=1,2, \ldots, n$, and $\left.\xi_{i, j} \in[0,1]\right)$, where $\xi_{i, j}$ denotes ponder value that is used by agent $i$ to ponder agent's $j$ assessment when calculating new trust values. In the extreme case when $\xi_{i, j}=0$ agent $i$ completely excludes agent's $j$ assessment from its calculations, while in case of $\xi_{i, j}=1$ agent $i$ takes into account assessment of agent $j$ as is.

Definition 4. A $k$-th trust vector is a $k$-th column in society assessment matrix A, i.e. $\mathbf{A}_{n, k}=$ $\left[\alpha_{1, k}, \alpha_{2, k}, \ldots, \alpha_{n, k}\right]$, while a $k$-th trust sub-vector is vector $\mathbf{A}_{n 1, k}=\left[\alpha_{1, k}, \alpha_{2, k}, \ldots, \alpha_{n 1, k}\right]$, where all unexisting ("-") values are excluded from the $k$-th trust vector, while the order of the remaining $n_{1}$ values remains unchanged.

Definition 5. QAD operators are $n$-ary functions $f_{i}\left(\alpha_{1, j}^{-}, \alpha_{2, j}^{-}, \alpha^{-}{ }_{3, j}, \ldots, \alpha_{n, j}^{-}\right)=\alpha^{+}{ }_{i, j}$, where $i$ denotes the $i$-th agent, superscript "-" denotes pre-operation value, superscript "+" postoperation value, and where $i=1,2, \ldots, n$. For the extreme optimistic operator (EOO), extreme pessimistic operator (EPO), centralistic consensus seeker (CCS), self-confident assessment operator $(\mathrm{CAO})$ and assessment hoping operator (AHO) the mappings are defined in Table 1 (note that $i, j=1,2, \ldots, n$ ):

Definition 6. Each agent society has associated a sequence of inclusiveness matrixes $\boldsymbol{I}_{1}, \ldots, \boldsymbol{I}_{k}$ for all $k$-steps of simulation. Each matrix consists of elements $i_{i, j} \in[0,1], i=1,2, \ldots, \mathrm{n}$ and $j=1,2, \ldots, \mathrm{n}$, where $\mathrm{t}_{i, j}=1$ means that $i$ discloses its assessment about agent $j$, while when $\mathrm{l}_{i, j}=0$ agent $i$ does not disclose its assessment of agent $j$.

This last definition is needed to take into account situations where agents may become non-cooperative and may intentionally stop disclosing their assessments of other agents, and 
Table 1. The main QAD operators

\begin{tabular}{|c|c|c|}
\hline$\alpha_{i, j}^{-} \neq-$ & $\max \left(\alpha_{1, j}^{-}, \alpha_{2, j}^{-}, \ldots, \alpha_{n 1, j}^{-}\right) \rightarrow \alpha_{i, j}^{+}$ & \\
\hline $\mathrm{EOO}$ & $\min \left(\alpha_{1, j}^{-}, \alpha_{2, j}^{-}, \ldots, \alpha_{n 1, j}^{-}\right) \rightarrow \alpha_{i, j}^{+}$ & \\
\hline $\mathrm{EPO}$ & $\left\{\begin{array}{l}{\left[\frac{1}{n_{1}} \sum_{k=1}^{n 1} \alpha_{\bar{k}, j} \rightarrow \alpha_{i, j}^{+}\right.} \\
\frac{1}{n_{1}} \sum_{k=1}^{n 1} \alpha_{k, j}^{-} \rightarrow \alpha_{i, j}^{+}\end{array}\right]$ & $\begin{array}{c}\text { if } \frac{1}{n_{1}} \sum_{k=1}^{n 1} \alpha_{k, j}^{-}<0 \\
\text { otherwise }\end{array}$ \\
\hline $\mathrm{CCS}$ & $\alpha_{i, j}^{-} \rightarrow \alpha_{i, j}^{+}$ & \\
\hline $\mathrm{CAO}$ & $\operatorname{rand}(-2,-1,0,1,2) \rightarrow \alpha_{i, j}^{+}$ & \\
\hline $\mathrm{AHO}$ & & \\
\hline$\alpha_{i, j}^{-}=-$ & $-\rightarrow \alpha_{i, j}^{+}$ & \\
\hline
\end{tabular}

vice-versa. In the text that follows it will be assumed that $\boldsymbol{I}_{1}=[1], \boldsymbol{I}_{2}=[1], \ldots, \boldsymbol{I}_{k}=[1]$, i.e. all agents are cooperative.

To put things into perspective let us demonstrate QAD use by applying it to the example society given in Figure 1. This society consists of four agents whose assessments are given in matrix $A_{1}$, while dependency matrix $\Xi$ consists solely of 1's, except the first column that consists only of 0 's. Further, let agent 2 be governed by extreme optimistic operator, agent 3 by extreme pessimistic operator, while agent 4 is governed by centralistic consensus seeker operator. Executing this society with the given values it turns out that the society gets into equilibrium in just one step. The corresponding assessments matrices are given in Eq. (1).

$$
A_{1}=\left[\begin{array}{cccc}
- & - & - & - \\
1 & - & 1 & - \\
- & 0 & -1 & - \\
-2 & - & 1 & 1
\end{array}\right] ; \quad A_{2}=\left[\begin{array}{cccc}
- & - & - & - \\
1 & - & 1 & - \\
- & 0 & -1 & - \\
0 & - & 0 & 1
\end{array}\right] .
$$

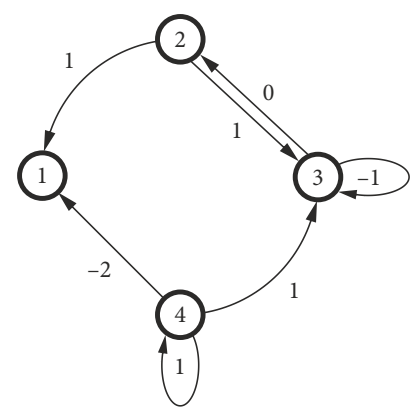

Figure 1. An example agents society with trust assessments 
In this simple example the system quickly gets into equilibrium. But if we included, for example, assessment hoping operator, the system would start to express various new forms that would have to be evaluated statistically. Similar would apply if inclusiveness matrices $\boldsymbol{I}_{k}$ would not equal [1] for all simulation steps, etc.

\subsection{Trust and agglomeration interplay dynamics}

In this subsection the interplay between agglomerations phenomena and trust will be studied. It will be assumed that a certain setting of agent society has been already simulated by using, e.g. QAD, so the final results of trust dynamics are available.

Suppose now we have nine agents that are positioned in a square with the first agent being in the upper left corner (north-west, NW, position), the second in the middle of the first row (north, $\mathrm{N}$, position), ..., and the last in the bottom right corner (south-east, SE, position). Let us assume further that after running ABM model with some QAD configuration the following assessment matrix $\mathrm{A}$ is obtained:

$$
A=\left[\begin{array}{ccccccccc}
2 & 1 & 1 & 1 & 0 & -2 & 0 & 1 & -2 \\
1 & 1 & 1 & 1 & 1 & 2 & 1 & 0 & 0 \\
1 & 2 & 2 & 2 & -1 & -1 & 2 & 1 & 1 \\
0 & 0 & 1 & 2 & -1 & 2 & 1 & 2 & 1 \\
0 & 2 & 0 & 1 & 2 & 1 & -2 & 1 & -1 \\
-1 & -1 & 0 & -1 & -1 & 2 & 2 & 2 & 2 \\
-1 & 1 & 0 & 1 & 2 & 1 & 2 & 0 & -2 \\
-2 & 1 & 1 & -1 & -1 & 1 & 1 & 0 & 2 \\
2 & 2 & 2 & -1 & -2 & 0 & -1 & -1 & 1
\end{array}\right] .
$$

Then the average trust values for agents, rounded to integer values, are as follows: NW:0, $\mathrm{N}: 1, \mathrm{NE}: 1, \mathrm{~W}: 1$, center:0, W:1, SW:1, S:1, SE:0.

Let's introduce now a simple fusion function. If an average trust value at an observed place is 2 , than the corresponding fused agglomeration phenomenon value is doubled. If the trust value equals 1 , then the fused agglomeration value remains the same, etc. The logic is that the initial agglomeration value is just multiplied by the corresponding trust value to obtain the final agglomeration.

Table 2. The initial agglomeration (left) and the trust fused agglomeration (right)

\begin{tabular}{|l|l|l|}
\hline 0.1 & 0.2 & 0.1 \\
\hline 0.2 & 0.1 & 0.2 \\
\hline 0.3 & 0.2 & 0.1 \\
\hline
\end{tabular}

\begin{tabular}{|c|c|c|}
\hline 0 & 0.2 & 0.1 \\
\hline 0.2 & 0 & 0.2 \\
\hline 0.3 & 0.2 & 0 \\
\hline
\end{tabular}

After applying the fusion function to the agglomeration situation that is given on the left of Table 2, we obtain the trust fused agglomeration on the right of Table 2. The basic agglomeration and the final one are also graphed in Figure 2. 


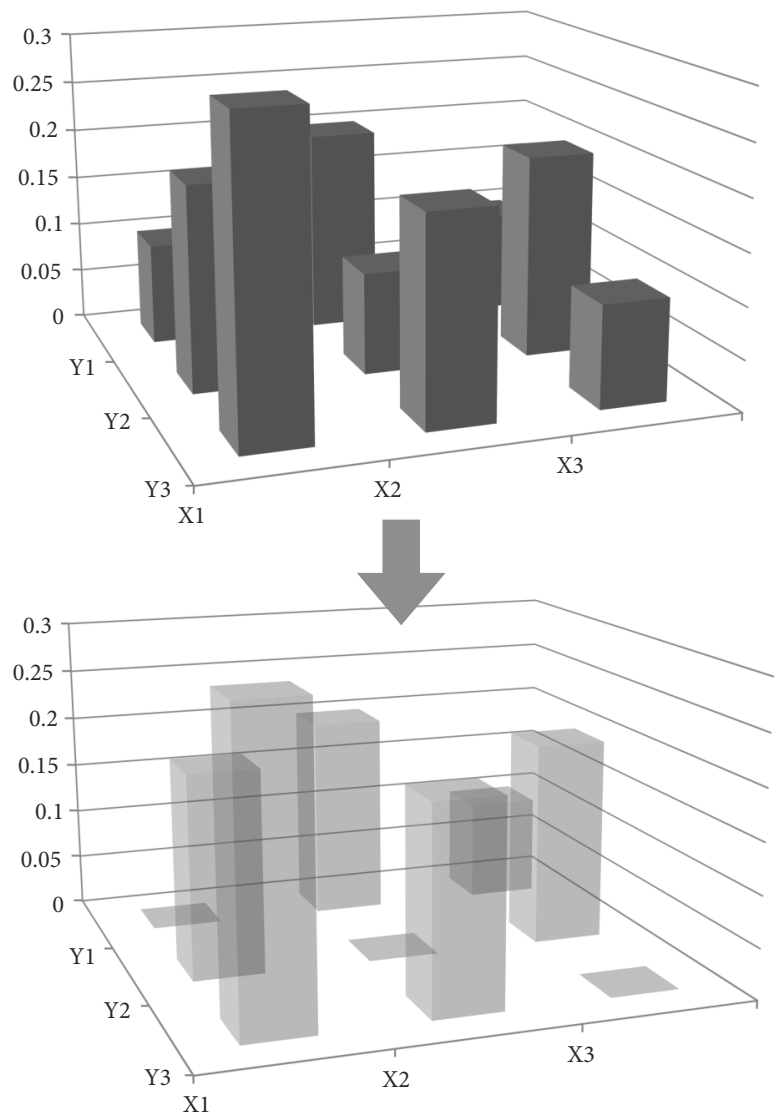

Figure 2. Trust fusion effects (the initial agglomeration is shown at the top, and trust fused agglomeration at the bottom)

\subsection{Discussion}

The above example is intentionally simplified for the sake of brevity and clarity. But it well represents how the developed apparatus operates, and what its strong points are. These are: bottom-up approach to analysis, inclusion of trust in economics phenomena, and if driven by real time data, the solution could enable simulations that faithfully reflect reality in close to real time manner.

Its weak point is that it requires conditions where tangible factors and trust can be considered as being independent, which consequently drive independently the evolution of the observed phenomenon. Another weak point is appropriate modelling of agents, because modelling humans with $\mathrm{ABM}$ is far from trivial. But even here some promising results in neuroscience research may provide solutions to this problem, especially when it comes to trust (Filkowski et al. 2016).

Evidently, the presented apparatus is the first step in the direction that is promising to expand the existing research possibilities, so its improvements are expected to follow. It is a 
common situation in science, including economics, that even decades old theoretical models have to be improved to meet real data (see, e.g. Cerina, Mureddu 2014; Mori, Smith 2015; Bode, Mutl 2010). This method will probably be no exception.

\section{Conclusions}

Trust is an important driver behind many economic activities. It is therefore surprising that it has not been studied more intensively in economics. This is despite the fact that its importance has been recognized in areas ranging from organizations level, where it is seen as a kind of a lubricant for successful business (Kramer, Tyler 1996; Bachmann 2006), to states level where it is seen as a key ingredient for prosperity of nations (Fukuyama 1995). Further, the traditional approaches to economics phenomena have been top-down, reflected in analytical models. But as H. M. Markowitz says: "If we restrict ourselves to models which can be solved analytically, we will be modelling for our mutual entertainment." Therefore, such methodological approaches are needed, which enable bottom-up research of economics phenomena, and which enable inclusion of trust.

This paper presents a bottom-up approach by linking areas of economics and computational trust management through ABM. By doing so it presents a new methodological approach, auxiliary composite simulations, which enables inclusion of trust in economics research areas, e.g. in study of spatial agglomerations.

The apparatus presented in this paper is expected to gain momentum in the near future thanks to technological advances in internet of things and big data analytics. These technologies will enable to measure agents' physiological signals and to obtain higher level signal aggregates in real time. By doing so these agents will be better modelled and driven by appropriate real-time data. This will result in simulations that will more faithfully reflect the reality. Consequently, we will be able to make another step towards better understanding and prediction of complex economics phenomena.

\section{Acknowledgements}

The presented research work has been enabled by Slovene Research Agency ARRS within the research program Pervasive computing, P2-0359. It also a partial result of cooperation in ISCH COST project IS1104 - The EU in the new complex geography of economic systems: models, tools and policy evaluation.

\section{Disclosure statement}

Author declares that he has no competing financial, professional, or personal interests from other parties. 


\section{References}

Anderson, L. R.; Mellor, J. M.; Milyo, J. 2006. Induced heterogeneity in trust experiments, Experimental Economics 9(3): 223-235. https://doi.org/10.1007/s10683-006-9124-2

Arthur, W. B. 2006. Out-of-equilibrium economics and agent-based modelling, in Handbook of computational economics vol. 2, 1551-1564. Amsterdam, North-Holland: Elsevier. https://doi.org/10.1016/S1574-0021(05)02032-0

Arthur, W. B.; Holland, H. H.; LeBaron, B.; Palmer, R.; Taylor, P. 1996. Asset pricing under endogenous expectation in an artificial stock market. Working Papers 96-12-093, Santa Fe Institute, December 1996.

Ashraf, N.; Bohnet, I.; Piankov, N. 2006. Decomposing trust and trustworthiness, Experimental Economics 9(3): 193-208. https://doi.org/10.1007/s10683-006-9122-4

Axtell, R. 2005. The complexity of exchange, The Economic Journal 115(504): F193-F210. https://doi.org/10.1111/j.1468-0297.2005.01001.x

Bachmann, R. 2006. Handbook of trust research. Cheltenham: Edward Elgar Publishing. https://doi.org/10.4337/9781847202819

Barrio, R. A.; Govezensky, T.; Ruiz-Gutiérrez, É; Kaski, K. K. 2017. Modelling trading networks and the role of trust, Physica A: Statistical Mechanics and its Applications 471: 68-79. https://doi.org/10.1016/j.physa.2016.11.144

Bode, E.; Mutl, J. 2010. Testing nonlinear new economic geography models, Economics Series 253. Vienna: Institute for Advanced Studies.

Calabuig, V.; Fatas, E.; Olcina, G.; Rodriguez-Lara, I. 2016. Carry a big stick, or no stick at all: Punishment and endowment heterogeneity in the trust game, Journal of Economic Psychology 57: 153-171. https://doi.org/10.1016/j.joep.2016.09.006

Cerina, F.; Mureddu, F. 2014. Is agglomeration really good for growth?, Journal of Urban Economics 84: 9-22. https://doi.org/10.1016/j.jue.2014.08.006

Cox, J. C.; Sadiraj, K.; Sadiraj, V. 2008. Implications of trust, fear, and reciprocity for modeling economic behaviour, Experimental Economics 11(1): 1-24. https://doi.org/10.1007/s10683-006-9156-7

Ferrin, D. L.; Dirks, K. T., 2003. The use of rewards to increase and decrease trust: mediating processes and differential effects, Organization Science 14(1): 18-31. https://doi.org/10.1287/orsc.14.1.18.12809

Filkowski, M. M.; Anderson, I. W.; Haas, B. W. 2016. Trying to trust: brain activity during interpersonal social attitude change, Cognitive, Affective, \& Behavioral Neuroscience 16(2): 325-338. https://doi.org/10.3758/s13415-015-0393-0

Fujita, M.; Krugman, P.; Venables, A. 1999. The spatial economy: cities, regions and international trade. New York: John Wiley.

Fukuyama, F. 1995. Trust: the social virtues and the creation of prosperity. New York: Free Press.

Jelenc, D.; Hermoso, R.; Sabater-Mir, J.; Trček, D. 2013. Decision making matters: a better way to evaluate trust models, Knowledge Based Systems 52: 147-164. https://doi.org/10.1016/j.knosys.2013.07.016

Johansson, B.; Quigley, J. M. 2004. Agglomeration and networks in spatial economics, Papers in Regional Science 83(1): 165-176. https://doi.org/10.1007/s10110-003-0181-z

Kahneman, D.; Tversky, A. 1979. Prospect theory: an analysis of decision under risk, Econometrica: Journal of the Econometric Society 47(2): 263-291. https://doi.org/10.2307/1914185

Keck, S.; Karelaia, N. 2012. Does competition foster trust? The role of tournament incentives, Experimental Economics 15(1): 204-228. https://doi.org/10.1007/s10683-011-9297-1

Keeley, B. 2007. Human capital: how what you know shapes your life. OECD, Paris. https://doi.org/10.1787/9789264029095-en 
Kimura, M. 2002. The use of agent-based models in regional science: $\mathrm{PhD}$ thesis. Cornell University.

Klos, T. B.; Nooteboom, B. 2001. Agent-based computational transaction cost economics, Journal of Economic Dynamics and Control 25(34): 503-526. https://doi.org/10.1016/S0165-1889(00)00034-8

Koesrindartoto, D.; Sun, J.; Tesfatsion, L. 2005. An agent-based computational laboratory for testing the economic reliability of wholesale power market, Power Engineering Society General Meeting, 1 August 2005, San Francisco, US.

Kramer, R. M; Tyler, T. R. 1996. Trust in organizations: frontiers of theory and research. Thousdand Oaks: SAGE Publications Incorporated.

Kurzban, R.; Rigdon, M. L.; Wilson, B. J. 2008. Incremental approaches to establishing trust, Experimental Economics 11(4): 370-389. https://doi.org/10.1007/s10683-007-9173-1

Leombruni, R.; Richiardi, M. 2003. Industry and labor dynamics: the agent-based computational economics approach, Proceedings of the Wild@ace 2003 Workshop, 3-4 October 2003, Torino, Italy. https://doi.org/10.1142/5706

Mori, T.; Smith, T. E. 2015. On the spatial scale of industrial agglomerations, Journal of Urban Economics 89: 1-20. https://doi.org/10.1016/j.jue.2015.01.006

Ortmann, A.; Fitzgerald, J.; Boeing, C. 2000. Trust, reciprocity, and social history: a re-examination, Experimental Economics 3(1): 81-100. https://doi.org/10.1023/A:1009946125005

Pessoa, L. 2008. On the relationship between emotion and cognition, Nature Reviews Neuroscience 9(2): 148-158. https://doi.org/10.1038/nrn2317

Putnam, R. D. 1995. Bowling alone: America's declining social capital, Journal of Democracy 6(1): 65-78. https://doi.org/10.1353/jod.1995.0002

Rosenthal, S.; Strange, W. 2008. The attenuation of human capital spillovers, Journal of Urban Economics 64(2): 373-389. https://doi.org/10.1016/j.jue.2008.02.006

Schotter, A.; Sopher, B. 2006. Trust and trustworthiness in games: an experimental study of intergenerational advice, Experimental Economics 9(2): 123-145. https://doi.org/10.1007/s10683-006-5386-y

Song, F. 2006. Trust and reciprocity in inter-individual versus inter-group interactions: the effects of social influence, group dynamics, and perspective biases, Experimental Economics 9(2): 179-180. https://doi.org/10.1007/s10683-006-7051-x

Tjosvold, D. 1985. Dynamics within participation: an experimental investigation, Group \& Organization Management 10(3): 260-277. https://doi.org/10.1177/105960118501000304

Trček, D. 2011. Trust management in the pervasive computing era, IEEE Security \& Privacy 9(4): 52-55. https://doi.org/10.1109/MSP.2011.95

Trček, D. 2012. An integrative architecture for a sensor-supported trust management system, Sensors 12(8): 10774-10787. https://doi.org/10.3390/s120810774

Trček, D. 2014. Qualitative assessment dynamics - complementing trust methods for decision making, International Journal of Information Technology \& Decision Making (IJITDM) 13(01): 155-173. https://doi.org/10.1142/S0219622014500072

Trichet, J. C. 2010. Reflections on the nature of monetary policy non-standard measures and finance theory, in Opening Address at the 6th ECB Central Banking Conference, 18 November 2018, Frankfurt am Main, Germany.

Zupančič, E.; Trček, D. 2017. QADE: a novel trust and reputation model for handling false trust values in e-commerce environments with subjectivity consideration, Technological and Economic Development of Economy 23(1): 81-110. https://doi.org/10.3846/20294913.2015.1022810

Zürn, M.; Topolinski, S. 2017. When trust comes easy: articulatory fluency increases transfers in the trust game, Journal of Economic Psychology 61(2017): 74-86. https://doi.org/10.1016/j.joep.2017.02.016 